Research Article

\title{
The Effect of Reynolds Number on Jet in Asymmetric Co-Flows: A CFD Study
}

\author{
Ziyan Teng $\mathbb{D}^{1},{ }^{1}$ I. A. Sofia Larsson, ${ }^{1}$ T. Staffan Lundström, ${ }^{1}$ and B. Daniel Marjavaara ${ }^{2}$ \\ ${ }^{1}$ Division of Fluid and Experimental Mechanics, Luleå University of Technology, Luleå SE-971 87, Sweden \\ ${ }^{2}$ Luossavaara-Kiirunavaara AB, Kiruna SE-981 86, Sweden \\ Correspondence should be addressed to Ziyan Teng; ziyan.teng@ltu.se
}

Received 2 May 2018; Revised 20 August 2018; Accepted 20 September 2018; Published 24 October 2018

Academic Editor: Evangelos Tsotsas

Copyright (c) 2018 Ziyan Teng et al. This is an open access article distributed under the Creative Commons Attribution License, which permits unrestricted use, distribution, and reproduction in any medium, provided the original work is properly cited.

In rotary kilns in grate-kiln systems for iron ore pelletizing, a long and stable jet flame is needed to ensure a high quality of the pellets. The primary jet issuing from the nozzle interacts with two asymmetric co-flows creating a very complex flow. In order to better understand and eventually model this flow with quality and trust, simplified cases need to be studied. In this work, a simplified and virtual model is built based on a down-scaled kiln model established in a previous experimental work. The aim is to numerically study the jet development as a function of position and Reynolds number (Re). The numerical simulations are carried out with the standard $k-\varepsilon$ model, and quite accurate velocity profiles are obtained while the centerline decays and spreading of the passive scalars are over predicted. The model is capable of predicting a Re dependency of the jet development. With increasing Re, the jet is longer while it generally decays and spreads faster resulting from the stronger shear between the jet and co-flows and the stronger entrainment from the recirculation zone. This recirculation found in the simulations restrain the momentum spreading in the spanwise direction, leading to a slower velocity spreading with higher Re. For further validation and understanding, more measurements in the shear layer and simulations with more advanced turbulence models are necessary.

\section{Introduction}

A grate kiln can be a part of an iron ore pellet sintering process, and it consists of a travelling grate, a rotary kiln, and a cooler. The rotary kiln can be about $35 \mathrm{~m}$ long and $6 \mathrm{~m}$ in diameter, and it carries iron ore pellets from the travelling grate to the cooler as the pellets are sintered at temperatures over $1000^{\circ} \mathrm{C}$. At the inlet of the rotary kiln, a burner is placed providing heat to the pellets. The flow from this burner can be modeled as a primary jet flow. Along with the primary jet, there are also two asymmetric co-flows from secondary air channels emanating from the cooler [1]. The two air channels provide excessive air for both the combustion and other important and earlier stages of the pelletizing process. As a result of the high flow rate and irregular shape of the air channels, the kiln performance is highly connected to the kiln aerodynamics, especially the development of the primary jet. The pelletizing process is complex since it is large scale and involves high temperatures and large amounts of air flowing in intricate geometries. Hence, it was realized early that relevant simplified cases should be studied to better understand the process and finally be able to model the whole process [2-10]. One of these cases have been studied both experimentally [8] and numerically [9] with a cold flow as often done for combustors, especially for diffusion flames where the combustion reaction is diffusion and mixing limited. One of the well-recognized reasons is that the cold flow structure is not significantly different from that with combustion reactions [11]. The early measurement by Glass and Bilger [12] indicated that a turbulent jet flame (or as they called "a heated jet-wake") and an isothermal turbulent round jet (isothermal jet-wake) have very similar turbulence quantities, such as the turbulence intensities and shear stress. Cold models provide an easy way to investigate the influence of different variables and facilitate measurements for validation [13]. This and the fact that the results 
can be compared to experiments, and previous simulations validate further studies on the simplified case in [2-6] now with focus on the jet development as a function of position and Reynolds number (Re).

Jet flow has been widely investigated from the beginning of the 1900s for its numerous applications. Great efforts have been put on the measurements of turbulent axisymmetric jets issuing into a quiescent environment in order to reveal the flow pattern (e.g. [14-18]). Due to the extensive applications in engineering problems, jets ejecting into moving co-flows are of great interest to researchers as well. The interactions between jets and the surrounding flows, which were named as "jet-wake" by Antonia and Bilger [19], make the flow field more complicated. The well-established selfpreserving property of turbulent axisymmetric jets moving into a fluid without any initial motion is no longer valid for jets in co-flows $[17,20]$. Research has focused on jets in axisymmetric co-flows, and the most studied cases are coaxial jets. Less is done on jets with asymmetric co-flows, despite the fact that they are also widely applied in industrial combustors.

It is nowadays well known that the initial conditions of a jet such as nozzle geometry and Re influence the development of the jet downstream. Since about four decades ago, the conclusion of a universal self-similarity behavior of all jets started to be challenged [21]. Boersma et al. [22] performed DNS simulations of jets with low Re and different initial velocity profiles. Their conclusion agrees with the earlier research by George [21] that there is no universal selfsimilarity among jets with different initial conditions. Mi et al. [23] reviewed early experimental research on turbulent round jets issued from different nozzles. They found that jets issuing from a smooth contraction nozzle have a larger spreading rate than jets issuing from a long straight round pipe. Moreover, the influence of the initial Re on the jet development in the self-similar far field depends on the type of the nozzle as well. The centerline decay of a passive scalar will increase with higher Re if the jet issues from a smooth contraction pipe, while a jet issuing from a long straight pipe is more independent of the initial Re.

By reviewing some of the experimental work regarding turbulent round jets with smoothly contracting jet nozzles with various Re ranges [18, 24-29], it can be concluded that there is no universal Re dependency of the jet mean centerline decay either. For jets with low Re, as stated in Todde et al. [24], the mean decay rate $1 / B$ and the virtual origin have different $\operatorname{Re}$ dependencies. The decay rate $1 / B$ decreases with $\mathrm{Re}$ until about $\mathrm{Re}=1600$, then remains constant until $\operatorname{Re}=4000$, and eventually increases again above 4000 . In other high Re cases, generally the decay term $B$ increases with $\mathrm{Re}$; that is, the decay rate $1 / B$ decreases, while above the threshold value $(\operatorname{Re}>20000$ as summarized in Zarruk and Cowen [29]), it is not significantly affected by Re.

The flames in the rotary kilns are confined diffusion jet flames with co-flowing air [5]. Co-flows, as well as the jet initial conditions, can significantly influence the jet development. As stated by Yule and Damou [30], the extraturbulence level brought by the co-flows will influence the spreading rate of the confined jet. Moreover, the co-flows in this work are neither annular nor coaxial to the primary jet. Thus, the high turbulence brought by the irregular co-flows in the rotary kiln may cause an unstable flow field, for example, extra-recirculation of reactants and products. The unstable flow field could lead to problems like combustion instability and thermal fatigue of kiln walls. A long and stable jet flame is needed for an even temperature profile within the pellet bed at the bottom of the kiln. To increase the combustion efficiency and lower the emission of pollutants such as $\mathrm{NO}_{\mathrm{x}}$, better mixing between the fuel (coal particles in the real kiln) and air is demanded. To reach these targets, it is necessary to know how to obtain a longer jet and better mixing in the kiln. The first option, based on the literature review of the previous research, is to change the jet initial condition by changing the geometry and see how the jet reacts to the changes.

The purpose of this work is to study the effect of initial Re, by altering the nozzle diameter $\left(D_{j}\right)$, on the far field development of the jet in a simplified cold model. The existence of the irregular-shaped secondary air channels brings an extra challenge compared to axisymmetric cases. Moreover, in the rotary kiln in this work, the air channels are separated by a surface called the "back plate." It covers $1 / 3$ of the kiln diameter, and the jet exit is located at the center. The co-flowing air streams flow over the back plate, which acts as a bluff body, and merge together downstream. Flow over bluff bodies are connected to vortex shedding. Previous experimental work by Larsson et al. [8] however showed that the vortex shedding behind the back plate was hard to detect when the jet was introduced. The results clearly show that the shedding process is present and that it breaks up the jet on a large scale and negatively affects the small scale mixing which is promoted by a stable shear layer. In the previous numerical study presented in ASME 2016 International Mechanical Engineering Congress and Exposition (IMECE 2016) by Teng et al. [9], different turbulence models were compared in simulating the interaction between the jet and the asymmetric coflows. It was found that the standard $k$ - $\varepsilon$ model performs the best in predicting the mean centerline decay and radial spreading of velocity and scalar concentration. Similar conclusions can be found in early work by Smith et al. [31] where the results of mean and RMS centerline decay and spreading of the jet from $k-\varepsilon$ models have good agreement with experimental data. In addition, the research work presented here on rotary kilns is highly linked to the industrial design, for which the robustness and computational economy are significant. Based on these facts, the standard $k-\varepsilon$ model was employed in this work to further investigate the effect of initial Re on the mean flow field in the down-scaled kiln model.

\section{Materials and Methods}

2.1. Geometry and Mesh. In previous research regarding kiln aerodynamics, a down-scaled, simplified rotary kiln model was established by Larsson et al. $[1,6,8]$. The rotary kiln was down scaled based on the Craya-Curtet parameter $C_{\mathrm{t}}=0.86$ as in the study by Larsson et al. [8], which represented a long 


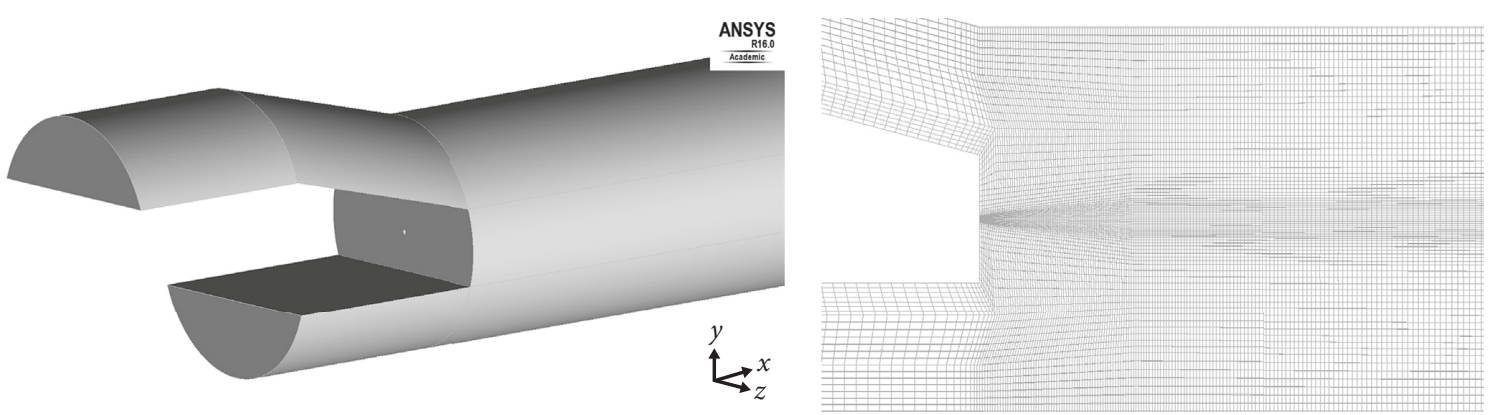

Figure 1: Geometry (part) of the down-scaled, simplified kiln model in Cartesian coordinate system and the hexagonal mesh (fine grid) with refinement near the jet inlet with multiple o-grids.

and lazy flame. In this work, the geometry as presented in Figure 1 was built based on this down-scaled kiln model within a Cartesian coordinate system. The $x$-axis represents the streamwise direction. The $y$ - and $z$-axis represent the spanwise directions. The kiln is simplified as a stationary cylinder with a diameter $D_{\text {kiln }}=0.1 \mathrm{~m}$, and the jet inlet is located in the center of the back plate with a diameter $D_{j}=$ $1.5 \mathrm{~mm}$. The height of the back plate is $1 / 3$ of the kiln diameter, and it separates the air channel into two parts. The upper secondary air channel has a $15^{\circ}$ inclining angle, and the lower one is parallel to the kiln. The kiln length is $1.5 \mathrm{~m}$.

As stated by Mi et al. [23] and Grandchamp et al. [32], the two commonly used nozzles, contraction nozzle, and straight round pipe, corresponding to a top-hat velocity profile and a parabolic velocity profile similar to the fully developed pipe flow, respectively. In this work, a top-hat velocity profile was approximated by a uniform mass flow rate at the jet inlet, since measurements of the initial velocity profile is lacking. To study the effect of initial Re, four cases were simulated with $D_{j}=$ $0.0012,0.0015,0.0018$, and $0.0021 \mathrm{~m}$ corresponding to $\operatorname{Re}=$ 37240, 29792, 24827, and 21280.

With the commercial meshing software ANSYS 16.0 ICEMCFD, a hexagonal mesh was built and multiple o-grids were applied to refine the part near the jet inlet (Figure 1). Extra attention was paid to the grid expansion perpendicular to the jet centerline to avoid too large aspect ratios and to ensure sufficient mesh quality. To enable a grid independence study, three grids with different refinement levels were prepared. The details of this study are presented in Section 2.5.

2.2. CFD Setup. The simulations were performed with the commercial code ANSYS CFX 16.0. Unsteady Reynoldsaveraged Navier-Stokes (uRANS) equations were used since steady state simulations oscillated regarding both the residuals and the monitored values. The time step was set to $1 \mathrm{~ms}$ based on the dimensionless time step $\Delta t U_{\mathrm{b}} / D_{\text {kiln }}=0.005$ used in Sofia Larsson et al. [6]. The simulation time was in its turn set to $5.5 \mathrm{~s}$ to ensure that the fluid would cover the entire kiln length, based on the bulk velocity. Transient statistics were taken from 5.5 to $7.5 \mathrm{~s}$ to obtain the time-averaged data.

The boundary conditions were the same as in Larsson et al. [8] and Teng et al. [9] with an even mass flow rate of $1.08 \mathrm{~kg} / \mathrm{s}$ at the two secondary channel inlets. The mass flow rate at the jet nozzle inlet was about $0.031 \mathrm{~kg} / \mathrm{s}$. At the kiln outlet, an average static pressure with a relative pressure of $0.0 \mathrm{~Pa}$ was applied. At the jet nozzle inlet, a passive scalar $(\Phi)$ was initialized as 1.0 while at the inlets of the two co-flows, it was 0.0 . The passive scalar helps to visualize the jet development and represents the mixing of the chemical species.

The advection scheme used was high resolution, which includes a blend factor that blends between first- and second-order accurate upwind schemes, ensuring a robust solution also in regions where the flow gradients change rapidly. A second-order backward Euler scheme was used to discretize the transient terms. The convergence criterion for the root-mean-square (RMS) residuals was $10^{-6}$ for tight convergence, and double precision was used. Each transient run was initialized with the corresponding steady state result.

2.3. Governing Equations. The continuity equation and the Navier-Stokes equations describing the mass and momentum transfer are

$$
\begin{aligned}
& \frac{\partial u_{i}}{\partial x_{i}}=0 \\
& \frac{D u_{i}}{D t}=-\frac{1}{\rho} \frac{\partial p}{\partial x_{i}}+v \frac{\partial^{2} u_{i}}{\partial x_{j}^{2}},
\end{aligned}
$$

where $x_{i}$ is the spatial coordinate, $p$ is the pressure, $t$ is time, and $v$ is the kinematic viscosity. The left-hand side in (2) is the material derivative of the velocity vector, $u_{i}$, of the flow field can be expanded as

$$
\frac{D u_{i}}{D t}=\frac{\partial u_{i}}{\partial t}+u_{j} \frac{\partial u_{i}}{\partial x_{j}}
$$

The Reynolds decomposition of the turbulent velocity field gives the mean-momentum or Reynolds equations based on the Navier-Stokes equations as

$$
\frac{\bar{D}\left\langle u_{i}\right\rangle}{\bar{D} t}=-\frac{1}{\rho} \frac{\partial\langle p\rangle}{\partial x_{i}}+v \frac{\partial^{2}\left\langle u_{i}\right\rangle}{\partial x_{j}^{2}}-\frac{\partial}{\partial x_{i}}\left\langle u_{i}^{\prime} u_{j}^{\prime}\right\rangle,
$$

where the brackets denote time-averaged values. Equation (4) can only be solved for very simple cases due to the 6 
components of the Reynolds stress tensor $u_{i}^{\prime} u_{j}^{\prime}$. These stresses consist of an isotropic and an anisotropic part according to

$$
\left\langle u_{i}^{\prime} u_{j}^{\prime}\right\rangle=\frac{2}{3} k \delta_{i j}+a_{i j},
$$

where $k(x, t)$ is the turbulent kinetic energy, $\delta_{i j}$ is the Kronecker delta, and $a_{i j}$ is the anisotropic part which, according to the specific assumption in the turbulentviscosity hypothesis, can be determined from

$$
a_{i j} \equiv\left\langle u_{i}^{\prime} u_{j}^{\prime}\right\rangle-\frac{2}{3} k \delta_{i j}=-v_{\mathrm{T}}\left(\frac{\partial\left\langle u_{i}\right\rangle}{\partial x_{j}}+\frac{\partial\left\langle u_{j}\right\rangle}{\partial x_{i}}\right),
$$

where $\nu_{\mathrm{T}}$ is the turbulent viscosity.

For visualization of the jet and to describe the concentration field, a passive scalar is introduced in the numerical model. The Reynolds-averaged scalar transport equation is

$$
\frac{\bar{D}\langle\Phi\rangle}{\bar{D} t}=\frac{\partial}{\partial x_{j}}\left(\left(D_{\Phi}+\frac{v_{\mathrm{t}}}{S c_{\mathrm{t}}}\right) \frac{\partial\langle\Phi\rangle}{\partial x_{j}}-\left\langle u_{i} \Phi\right\rangle\right)+\left\langle S_{\Phi}\right\rangle,
$$

where $\Phi$ is the quantity of the passive scalar. It could for example be the concentration of a species. $\Phi^{\prime}$ is the fluctuating scalar field, and $D_{\Phi}$ is the molecular diffusivity, which is not considered in this nonreactive case since the molecular diffusion has quite little effect on the scalar transport compared to the convection. $v_{t} / S c_{t}$ is the turbulent diffusivity, in which $\nu_{\mathrm{t}}$ is the turbulent eddy viscosity, and $\mathrm{Sc}_{\mathrm{t}}$ is the turbulent $\mathrm{Schmidt}$ number. $\mathrm{Sc}_{\mathrm{t}}$ describes the ratio between the turbulent diffusivity and the molecular diffusivity of mass. In this work $\mathrm{Sc}_{\mathrm{t}}$ is set as a constant 0.7 based on research work regarding axisymmetric free jet flows by Tominaga and Stathopoulos [33] and Mejía et al. [34]. $S_{\Phi}$ represents the Reynolds-averaged source term, and in this nonreactive case, the source term is zero.

2.4. Turbulence Models. The standard $k-\varepsilon$ model is widely used in industrial problems for its robustness. In previous work by Granström et al. [10] and Teng et al. [9], the standard $k-\varepsilon$ model was proved to predict the mean flow field in the kiln in a proper way. Since the detailed structures of the jet, especially the small scale flow motions, is not taken into account in this work, the standard $k-\varepsilon$ model, again, was used to close the governing equations. The $k$ - $\varepsilon$ model solves two equations for turbulent kinetic energy $k$ and the energy dissipation rate $\varepsilon$ :

$$
\begin{aligned}
& \frac{\bar{D} k}{\bar{D} t}=\frac{\partial}{\partial x_{j}}\left(\frac{\mu_{\mathrm{t}}}{\sigma_{k}} \frac{\partial k}{\partial x_{j}}\right)+2 \mu_{\mathrm{t}} E_{i j} E_{i j}-\rho \varepsilon, \\
& \overline{\bar{D} \varepsilon} \\
& \overline{\bar{D} t}=\frac{\partial}{\partial x_{j}}\left(\frac{\mu_{\mathrm{t}}}{\sigma_{\varepsilon}} \frac{\partial \varepsilon}{\partial x_{j}}\right)+C_{\varepsilon 1} \frac{\varepsilon}{k} 2 \mu_{\mathrm{t}} E_{i j} E_{i j}-C_{\varepsilon 2} \rho \frac{\varepsilon^{2}}{k},
\end{aligned}
$$

where $E_{i j}$ represents the component of rate of deformation. The turbulent eddy viscosity $\mu_{\mathrm{t}}$ is specified as

$$
\mu_{\mathrm{t}}=\frac{\rho C_{\mu} k^{2}}{\varepsilon} .
$$

The standard $k-\varepsilon$ model is typically referred to that developed by Launder and Sharma [35]. The model constants used are

$$
C_{\mu}=0.09, C_{\varepsilon 1}=1.44, C_{\varepsilon 2}=1.92, \sigma_{\kappa}=1.0, \sigma_{\varepsilon}=1.3 .
$$

2.5. Grid Study. To perform the grid independence study, three grids were prepared. The grid refinement factor calculated based on the procedure in Celik [36] was about 1.3. As mentioned in Section 2.1, the mesh was built by a structured mesh with multiple o-grids in order to refine the grid near the jet inlet. The refinement was done in a structured way to ensure a systematic discretization error estimation.

Two variables were chosen for the error estimation, the time-averaged scalar concentration at $60 D_{j}$ on the geometrical centerline, and the radial spreading rate $S$ calculated based on the jet half width $r_{1 / 2}(x)$ from 0 to $100 D_{j}$.

The jet half width $r_{1 / 2}(x)$ is defined as the spanwise location where the mean velocity or scalar concentration equals to half of the jet centerline value. Ideally, a round jet develops symmetrically, and $r_{1 / 2}(x)$ should be isotropic at each YOZ plane. For a self-preserving jet, $r_{1 / 2}(x)$ has a linear relationship with the streamwise location $x$ as follows [18]:

$$
\frac{r_{1 / 2}(x)}{D_{j}}=S\left(\frac{x-x_{0}}{D_{j}}\right) \text {. }
$$

The term $S$ is the spreading rate for momentum (velocity) or a passive scalar in the spanwise direction. To distinguish between them, $S$ denotes the spreading rate of momentum, and $S_{\Phi}$ denotes the spreading rate of a passive scalar. At the right-hand side, $x$ is the streamwise location of the observation point, and $x_{0}$ is the location of the virtual origin. For ideal cases, $S$ and $S_{\Phi}$ are treated as constants, and they can be calculated from (11) based on $r_{1 / 2}$. The spreading rates represent how fast the jet spreads in the spanwise direction. The larger spreading rates a jet has, the faster it spreads, and as a result, the better it mixes with the surrounding flow. Therefore, spreading rates are often used to describe the development of a turbulent round jet in the spanwise direction.

It is worth to note that, from previous simulations by Teng et al. [9], it was found that the jet develops asymmetrically because of the geometry. As a result, the jet half width $r_{1 / 2}(x)$ is no longer isotropic. The visualization of the velocity fields where the streamwise velocity $U \geq U_{\mathrm{c}} / 2$ from the steady state simulation of the case with $D_{j}=0.0015 \mathrm{~m}$ clearly shows an asymmetric jet development downstream (Figure 2). $U_{\mathrm{c}}$ is the jet centerline velocity at each streamwise location. As the jet develops, the shapes of the velocity contours are nearly elliptical, and they are defined as the jet 


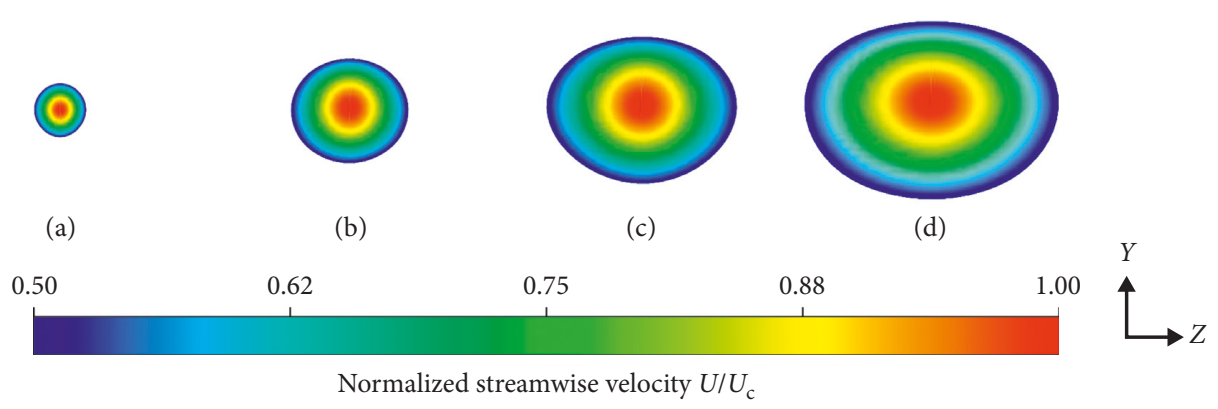

Figure 2: Jet half width regions at $x=$ (a) 30, (b) 60, (c) 90, and (d) $120 D_{j}$ from steady state simulation with $D_{j}=0.0015 \mathrm{~m}$.

half width regions in this work. Moreover, they are nonaxisymmetric as depicted in Figure 2. Therefore, in order to quantify the jet spreading in the spanwise direction, the jet half widths in this work are estimated by the areas of these jet half regions taken every $2 D_{j}$ downstream in the $X$-direction.

Richardson extrapolation was performed based on the procedure described by Celik [36] using the results from steady state simulations with three grids of different densities. The grid information and the Richardson extrapolation results show an oscillatory convergence with these three girds (Table 1). The errors between the extrapolated value and the simulated values, $e_{\text {ext, } 1}$ and $e_{\text {ext, } 2}$, respectively, are quite small. It is not necessary to always use the finest mesh as long as the errors between the results with the second grid and the extrapolated value are small enough. In this work, the second grid was therefore chosen for further investigation to save computational time.

\section{Results and Discussion}

To investigate the effect of initial Re on the jet development, four simulations with different nozzle diameters $D_{j}=0.0012$, $0.0015,0.0018$, and $0.0021 \mathrm{~m}$ were run with grid 2 based on the grid study results. With the same initial mass flow rate $0.031 \mathrm{~kg} / \mathrm{s}$ as in the PIV measurement by Larsson et al. [8], the jet initial velocities are $U_{j}=27.70,17.73,12.31$, and $9.04 \mathrm{~m} / \mathrm{s}$, respectively, for the four cases. The corresponding initial Re are 37240, 29792, 24827, and 21280. Among these four cases, the one with $D_{j}=0.0015 \mathrm{~m}(\operatorname{Re}=29792)$ is seen as the standard case since it is directly built based on the work in [8].

The instantaneous streamwise velocity fields (Figure 3 ) at the last time step show the major difference brought by altering the nozzle diameter $D_{j}$. In order to visualize the jet in a better way, the streamwise velocity is normalized as $U / U_{\mathrm{b}} . U_{\mathrm{b}}$ is the bulk velocity in the whole kiln calculated by the total flow rate and the kiln cross-sectional area. The high-velocity region where $U / U_{\mathrm{b}}>5$ can represent the jet length, and it increases with Re, which is beneficial to the sintering of pellets. On the contrary, the length of the region where $1<U / U_{\mathrm{b}}<5$ significantly decreases with Re, indicating that the velocity decays faster in higher Re cases. This region can be called the fast-decay region. It can be seen that a low-velocity region where $U / U_{\mathrm{b}}<1$ forms at the upper kiln wall after the upper co-flow starts to interact with the primary jet in all cases. This low-velocity region orients the jet by pushing it downwards, restraining the jet from spreading upwards. Inside this region, recirculation
TABLE 1: Grid information and Richardson extrapolation results with three grids and steady state simulations.

\begin{tabular}{lccc}
\hline & $\begin{array}{c}N_{\text {element }} \\
\text { (million) }\end{array}$ & $\Phi_{0,60_{D_{j}}}$ & $S$ \\
\hline Grid 3 (coarse) & 1.0 & 0.07660 & 0.1120 \\
Grid 2 (medium) & 2.4 & 0.07688 & 0.1115 \\
Grid 1 (fine) & 5.5 & 0.07674 & 0.1116 \\
Extrapolated value $(\mathrm{h} \rightarrow 0)$ & & 0.07660 & 0.1116 \\
$e_{\text {ext,1 }}$ & & $0.18 \%$ & $0.04 \%$ \\
$e_{\text {ext,2 }}$ & & $0.36 \%$ & $0.15 \%$ \\
\hline
\end{tabular}

may happen. The recirculation is marked by black contour lines in Figure 3 to visualize the location and area of the back flow, which is the region where the flow moves in the negative $X$-direction.

From the turbulent kinetic energy profile taken at $x=$ $0.09 \mathrm{~m}$ (corresponding to $60 D_{j}$ in the standard case), the existence and location of the recirculation zone disclosed in Figure 3 can be identified as well. In Figure 4, the turbulence kinetic energy is first scaled by the square of centerline velocity $U_{\mathrm{c}}$ at $x=0.09 \mathrm{~m}$ (Figure $4(\mathrm{a})$ ) and then expressed in the logarithm scale (Figure 4(b)) to highlight the region where the weak co-flows affect the flow field. The $x$-axis in Figure 4 is the normalized turbulence kinetic energy. The $y$ axis is the spanwise distance $y$ from the jet centerline in $Y$ direction. The profiles of the kinetic energy depict that there is a large scale flow motion outside the jet, though it is weaker quantitatively compared to the jet itself. It corresponds to the upper recirculation zone seen in Figure 3. With increasing Re (decreasing $D_{j}$ ), the boundary of the recirculation zone moves inwards to the jet centerline in the $Y$-direction and the turbulence kinetic energy increases, indicating that the recirculation zone is enlarged and enhanced.

In combustion reactors, there are two types of recirculation zones: the internal recirculation zone (IRZ) and the external recirculation zone (ERZ). They are of great significance for diffusion flames since they directly influence the mixing between the fuel and oxidizer. The IRZ is located inside the jet and has been proven to be able to improve the internal mixing and flame stability according to Driscoll et al. [37] and Vanoverberghe et al. [38] for instance. The ERZ is located outside the jet, and it can be generated by shear and the entrainment of ambient flow by the jet in a confined environment. Rabacal et al. [39] 


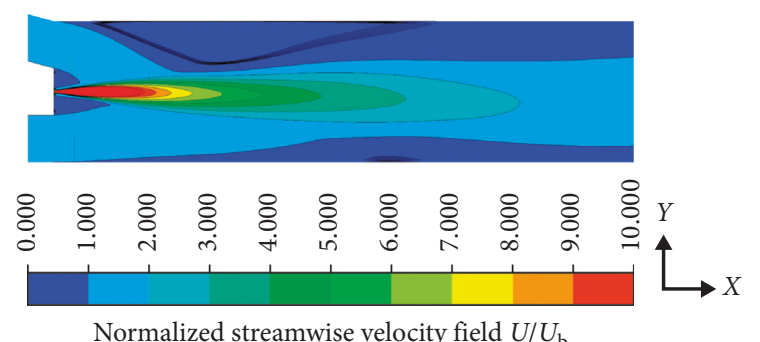

(a)

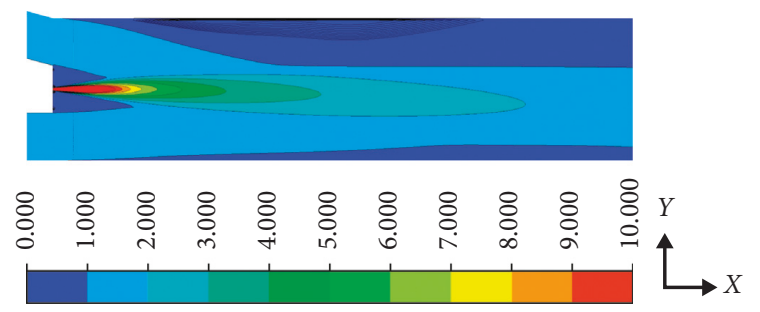

Normalized streamwise velocity field $U / U_{\mathrm{b}}$

(c)
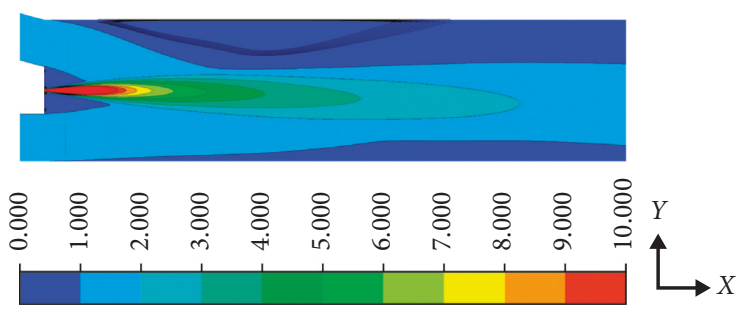

Normalized streamwise velocity field $U / U_{\mathrm{b}}$

(b)
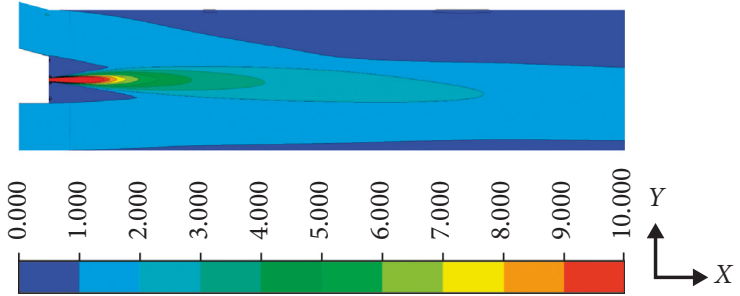

Normalized streamwise velocity field $U / U_{\mathrm{b}}$

(d)

FIgURE 3: Instantaneous streamwise velocity contours at 5.5 seconds on the symmetry plane XOY: (a) $\operatorname{Re}=37240 ;(b) \operatorname{Re}=29792 ;(c) \operatorname{Re}=$ 24827; (d) $\operatorname{Re}=21280$.

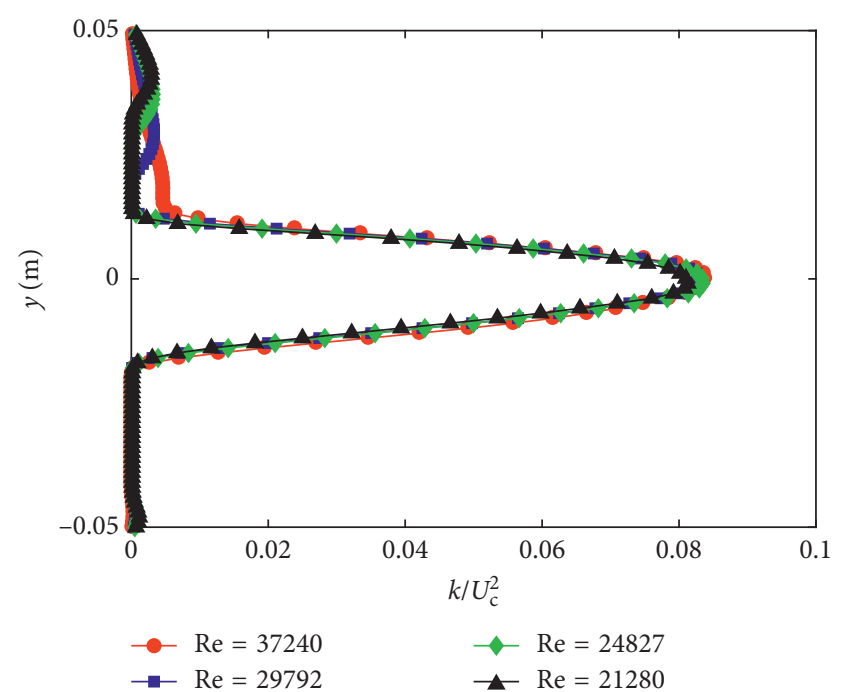

(a)

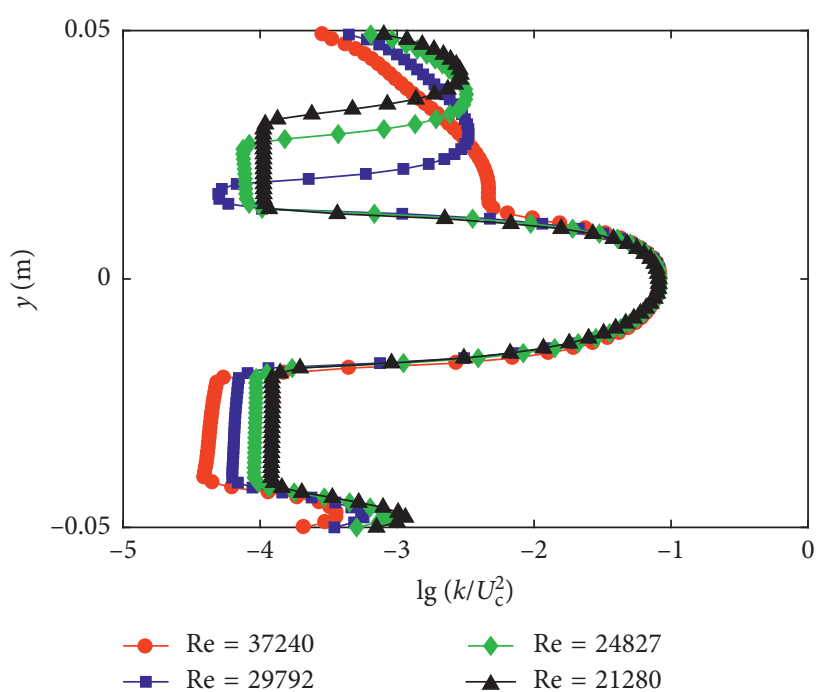

(b)

Figure 4: Normalized turbulence kinetic energy profiles on the vertical centerline at $x=0.09 \mathrm{~m}$.

concluded that, based on large eddy simulation (LES) results of a pulverized coal jet flame, the reactant particles recirculate inside the ERZ until being fully burned. As a consequence, the ERZ may increase the residence time and the burnout rate of the fuel particles and then further affects the flame stability.

In this work, the four cases are nonreactive and singlephase, and hence, the passive scalar field is used to show the effect of the ERZ formed near the upper kiln wall on mixing. A passive scalar is a diffusive contaminant without dynamic effect on the flow motion. The transport of a passive scalar is governed by Equation (7), in which the diffusion term consists of turbulent and molecular diffusion. The distribution of the passive scalar can represent the mixing and entrainment of species and can be useful for the understanding of the mixing status in a fluid flow.

It can be seen from the scalar concentration field (Figure 5) that, at the same location as the ERZ shown in Figure 3, the passive scalar is entrained up towards the upper kiln wall. It indicates that the appearance of the ERZ promotes the spreading of the passive scalar in the spanwise direction in the upper part of the kiln. In the reactive case, the ERZ can improve the mixing between the reactants, assist in the flame-anchoring, and further benefit 


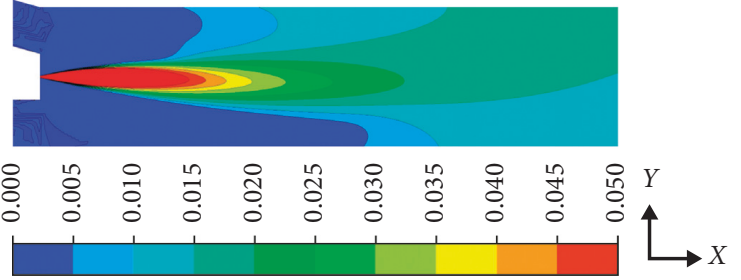

Time-averaged scalar concentration (specific) $\bar{\Phi}$

(a)

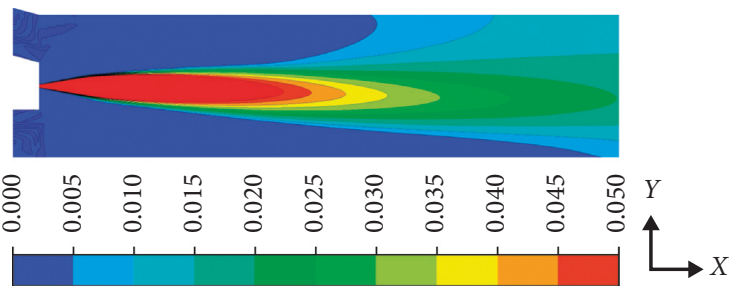

Time-averaged scalar concentration (specific) $\bar{\Phi}$

(c)

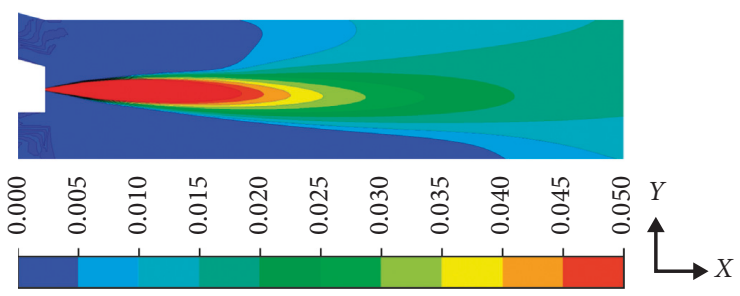

Time-averaged scalar concentration (specific) $\bar{\Phi}$

(b)

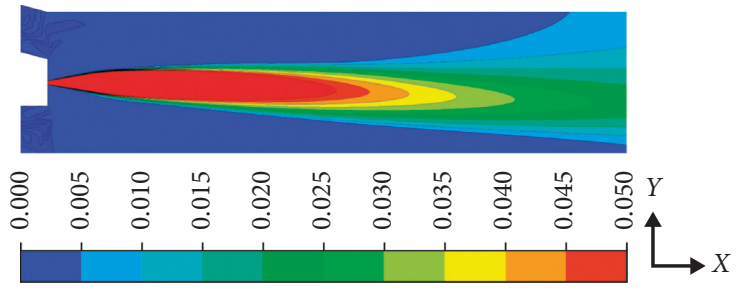

Time-averaged scalar concentration (specific) $\bar{\Phi}$

(d)

Figure 5: The time-averaged passive scalar fields changing with $\operatorname{Re}$ on the symmetry plane XOY: (a) $\operatorname{Re}=37240 ;$ (b) $\operatorname{Re}=27972 ;(c) \operatorname{Re}=$ 24827; (d) $\operatorname{Re}=21280$.

the flame stability. However, the ERZ orients the jet downwards, which may bring an uneven temperature field to the kiln bed. As disclosed in Figures 3-5, with decreasing Re, the ERZ is considerably weakened until it almost disappears. The effect from the ERZ is then almost eliminated.

Besides the spreading rate mentioned earlier, another important characterization of the mean flow field of a turbulent round jet is the centerline decay. The decay of the mean velocity of such a jet at the centerline $U_{c}$, with the streamwise distance from the nozzle $x$, can be expressed with a linear relationship as in [18]:

$$
\frac{U_{j}}{U_{c}}=\frac{1}{B}\left(\frac{x-x_{0}}{D_{j}}\right) .
$$

Similarly, it has been suggested that the decay of the centerline concentration $\Phi_{c}$ of a turbulent round jet can be written as in [23]:

$$
\frac{\Phi_{j}}{\Phi_{c}}=\frac{1}{B_{\Phi}}\left(\frac{x-x_{0}}{D_{j}}\right) .
$$

The terms $1 / B$ and $1 / B_{\Phi}$, the decay rates of momentum (velocity), and the passive scalar, for ideal cases, are treated as constants. $U_{j}$ is the jet initial velocity, and $\Phi_{j}$ is the passive scalar concentration at the jet inlet, which was defined as 1 in the mass fraction form in this work.

The decay rates of the mean streamwise velocity $1 / B$ and the scalar concentration $1 / B_{\Phi}$ are calculated by the curve fitting the time-averaged data from simulation results based on Equations (12) and (13). For validation, data from 30 to $50 D_{j}$ were used for calculation in order to be consistent with Larsson et al. [8]. From the profiles of $U_{j} / U_{c}$ and $\Phi_{j} / \Phi_{c}$ shown in Figure 6, it can be seen that, for $x<6 D_{j}$, there is no decay for any Re. This is the so-called potential core of the jet with a virtually uniform mean velocity. The decay rate of the mean streamwise velocity $1 / B$ increases with Re (Figure 6(a)), while $1 / B_{\Phi}$ of the mean scalar is not significantly affected (Figure 6(b)). This independency of Re of the mean scalar decay agrees with the findings in the coupled PIV-LIF measurements by Zarruk and Cowen [29]. It is also clear that $1 / B_{u}$ is dependent on $x$ for all Re investigated, and hence, $1 / B_{u}=f_{1}(\operatorname{Re}, x)$.

The spreading rates of the streamwise velocity $S$ and the passive scalar concentration $S_{\Phi}$ in the spanwise direction are calculated based on Equation (11). As described in Section 2.5, the jet half widths $r_{1 / 2}$ are estimated from the areas of jet half width regions taken from the time-averaged data. From Figure 7, it can be seen that the spreading rate of velocity $S$ slightly decreases with Re (Figure 7(a)), but it almost remains constant. The spreading rate of the scalar $S_{\Phi}$ has an obvious different trend in that it increases with Re (Figure $7(\mathrm{~b})$ ), meaning that $S_{\Phi}=f_{2}(\operatorname{Re}, x)$. In addition, a slower increase of $S_{\Phi}$ is seen after about $25 D_{j}$ in all cases, indicating that the scalar spreads slower. This agrees with Larsson et al. [23], who concluded that there is less mixing after the wake region extending to about $x / D_{j}=25$. With higher $\operatorname{Re}\left(\right.$ smaller $\left.D_{j}\right)$, this effect tends to be eliminated.

All the terms calculated from the centerline decay and spreading are summarized in Table 2 together with the experimental data from Larsson et al. [8]. A comparison of the simulation results to the measurement shows that the standard $k-\varepsilon$ model overpredicts the centerline decay term $B$ by about $45 \%$ and the spreading rate of scalar concentration $S_{\Phi}$ by about $20 \%$. The prediction of the spreading rate of the velocity $S$ is relatively accurate with an error of about $3 \%$. The $k-\varepsilon$ models can be improved for better prediction of the centerline decay and spreading of turbulent round jets by modifying the model constants based on individual decay and spreading data of each jet [38]. 


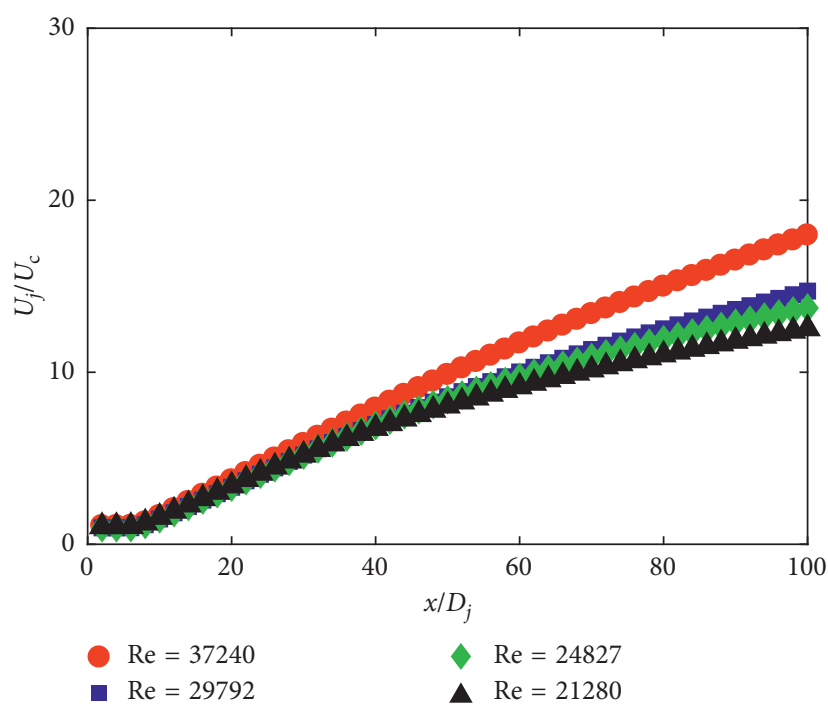

(a)

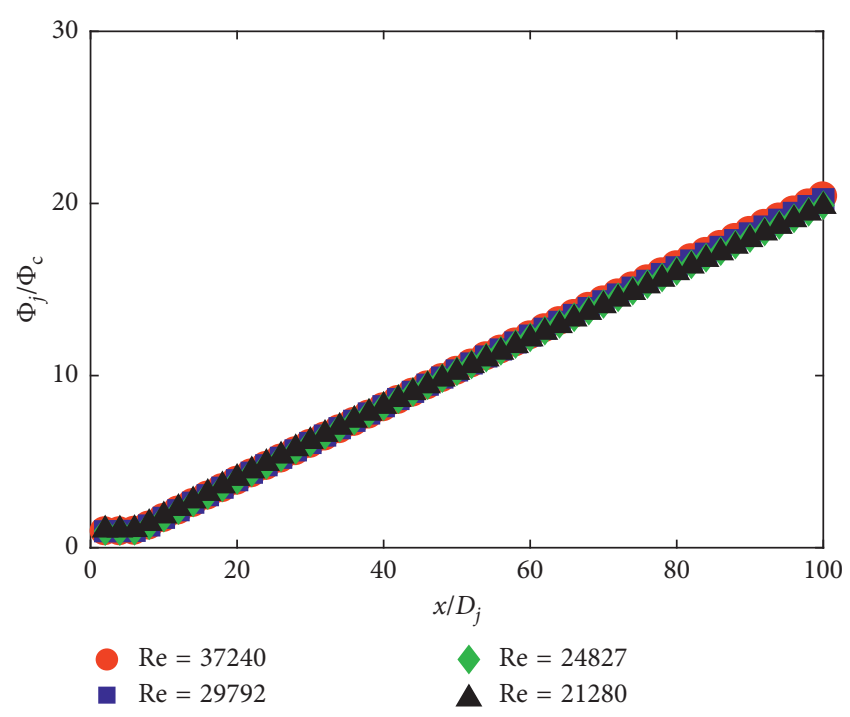

(b)

FIgURE 6: The centerline decay of the mean: (a) streamwise velocity; (b) scalar concentration.

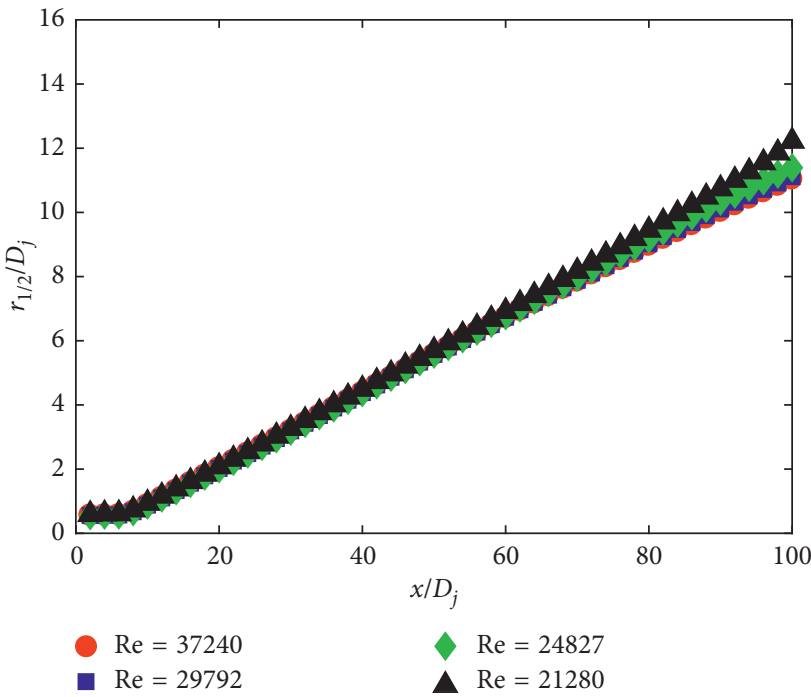

(a)

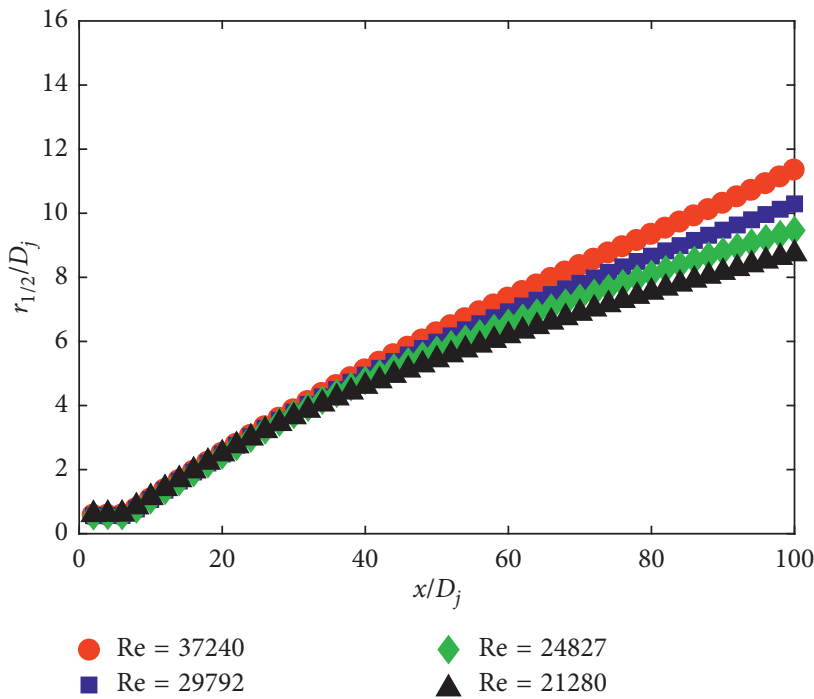

(b)

FIgURE 7: The calculated spreading of the mean: (a) streamwise velocity; (b) scalar concentration.

TABLE 2: The calculated centerline decay and radial spreading terms.

\begin{tabular}{lcccccccc}
\hline \multirow{2}{*}{$\begin{array}{c}\text { Re } \\
\end{array}$} & This work & Larsson et al. [8] & This work & Larsson et al. [8] & This work & Larsson et al. [8] & This work & \multicolumn{2}{c}{$S_{\Phi}$} \\
& Larsson et al. [8] \\
\hline 21,280 & 6.58 & - & 5.02 & - & 0.121 & - & 0.089 & - \\
24,827 & 6.19 & 4.25 & 4.95 & - & 0.120 & 0.124 & 0.100 & 0.083 \\
29,792 & 6.03 & - & 4.92 & - & 0.119 & - & 0.109 & - \\
37,240 & 5.22 & - & 4.92 & - & 0.118 & - & 0.120 & - \\
\hline
\end{tabular}

Zarruk and Cowen [29] also concluded that the decay term $B$ of the mean velocity increases with initial Re and then tends to be stable at very high $\operatorname{Re}(>20,000)$ by reviewing the studies of turbulent round jets without co-flow. An opposite trend is seen in this work where $B$ decreases with Re. There are several possible explanations to the discrepancy in $\mathrm{Re}$ dependency of mean centerline decay and spreading between this work and those studies without co-flow. Firstly, in this 


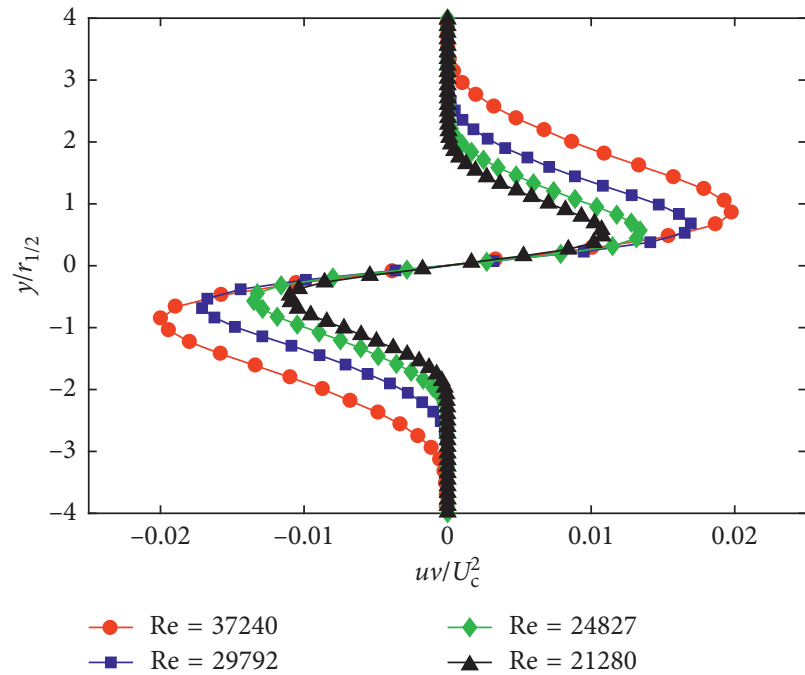

(a)

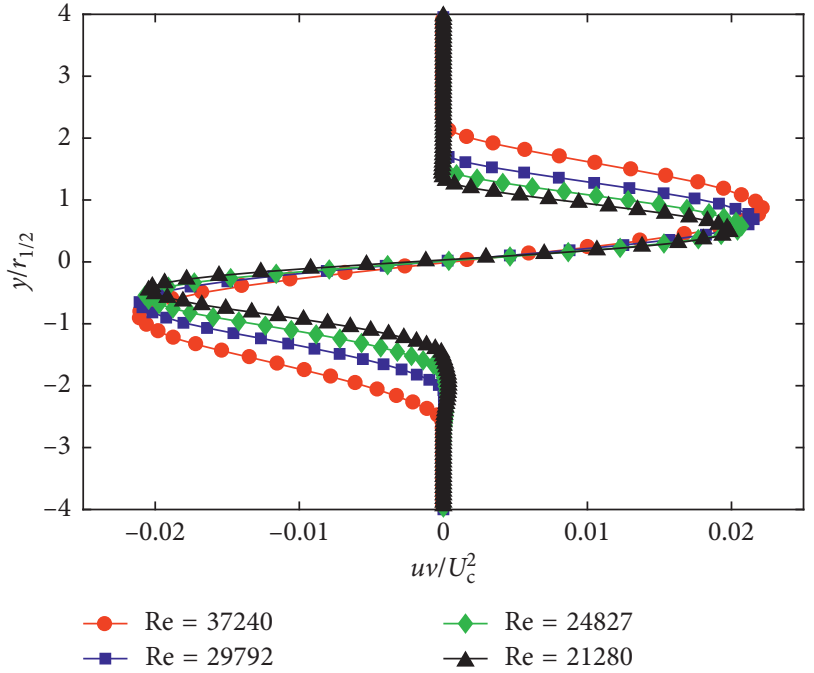

(b)

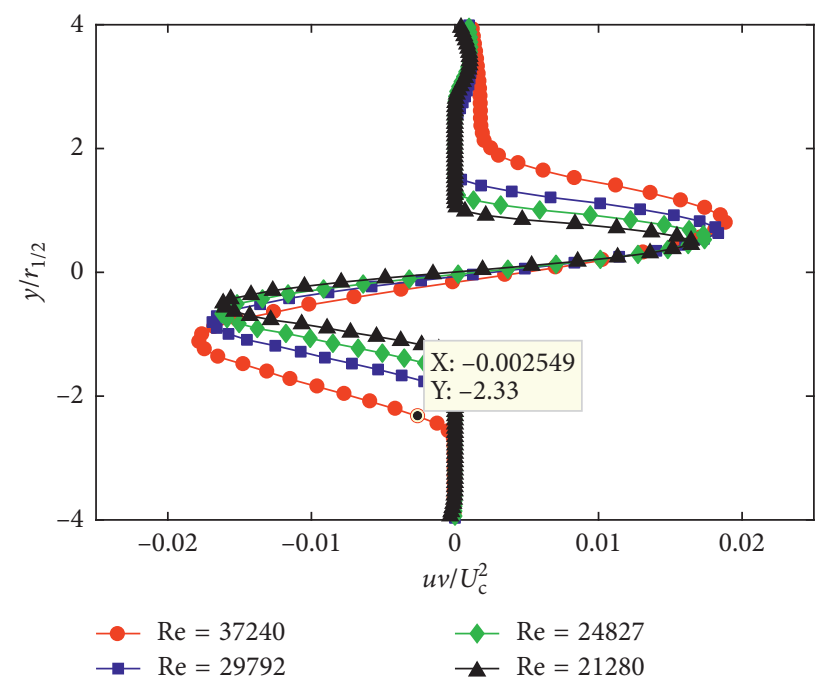

(c)

Figure 8: The profile of shear stress $\overline{u v} / U_{\mathrm{c}}^{2} \sim y / r_{1 / 2}$ at (a) $x=0.015 \mathrm{~m}$, (b) $x=0.045 \mathrm{~m}$, and (c) $x=0.09 \mathrm{~m}$ (corresponding to 10,30 , and $45 D_{j}$ in the standard case) in the $Y$-direction.

work, there are two co-flows with an asymmetric, nonannular geometry, and a back plate separating them. When Re increases, the velocity difference between the jet and the coflows increase as well, resulting in stronger shear between them. Entrainment of the surrounding flows into the primary jet is promoted by the shear layers at the interfaces between the jet and co-flows formed by the large velocity gradient there. Strong shear can be identified from the radial profile of the shear stress $\overline{u v}$ taken at $x=0.015,0.045$, and $0.09 \mathrm{~m}$ (Figure 8). The $x$-axis, stress $\overline{u v}$, is scaled by the square of the centerline velocity $U_{c}$ at each streamwise location. The $y$-axis, the spanwise distance from the centerline $y$, is scaled by the jet half widths calculated previously. It can be seen that, with increasing $\mathrm{Re}$, the shear layers are stronger and located more outwards in the spanwise direction. In other words, the jet is wider with higher Re. In addition, it is found before that the ERZ formed near the upper kiln wall suppresses the jet downwards and this zone is enlarged and reinforced at higher Re. At the same time, it entrains the passive scalar upwards. In other words, the ERZ restrains the momentum spreading while it prompts the scalar spreading in the spanwise direction.

In earlier work by Larsson et al. [5], it was found that the back plate between the two co-flows causes vortex shedding. Furthermore, in the measurements by Larsson et al. [8], the vortex shedding behind the back plate was hard to detect when the primary jet was introduced. The results clearly shows that the shedding process is present and that it breaks up the jet on a large scale and negatively affects the small scale mixing which is promoted by a stable shear layer. However, the effect from such underlying flow structures cannot be modeled by $k-\varepsilon$ models. This might be another possible reason for the opposite trend of the mean flow field dependence on Re. Two-equation models are not capable of predicting anisotropy and different turbulence 
length scales. As stated in Smith et al. [40], even though $k-\varepsilon$ models perform better than other two-equation models in predicting the spreading rate of round jets, the underlying flow structures are not taken into account. It results in failure of predicting the correct trends of the centerline decay rate of jets with different nozzle geometries. This problem might be solved by finding suitable model constants for jets with different initial conditions where the constants are functions of the centerline decay rates and the jet half widths [41]. Despite the fact that the spreading issue can be taken care of, $k-\varepsilon$ models still cannot capture the effect from vortex shedding. This implies that more advanced turbulence models are necessary, as well as more measurements, for a more thorough understanding of how the jet initial conditions affect the flow field. This work including the published prestudy [9] are well summarized in thesis [42].

\section{Conclusions}

Based on the results from CFD simulations with the standard $k-\varepsilon$ model and comparisons to other research work, some conclusions can be drawn as below.

The standard $k-\varepsilon$ model gave the closest prediction to the measurements in previous work. The prediction of the spreading rate $S$ of the streamwise velocity is quite accurate with an error of about $3 \%$. There is an overprediction of the centerline decay term $B$ of the velocity by about $45 \%$ and of the spreading rate $S_{\Phi}$ of the passive scalar by about $20 \%$ compared to the results in previous measurements. The model is capable of predicting a Re dependency of the jet development as the jet with higher initial Re is longer, which is beneficial to the sintering of pellets, but the centerline velocity decays faster downstream. The spanwise spreading rate $S_{\Phi}$ of the passive scalar increases with $\mathrm{Re}$, but the spreading rate $S$ of the velocity almost remains constant when Re increases. One of the reasons behind this Re dependency could be the enhanced shear layer between the jet and co-flows resulting from higher velocity difference when Re increases. This stronger shear also improves the scalar mixing in the flow field.

An external recirculation zone (ERZ) forms at the upper kiln wall after the inclined upper co-flow and starts to interact with the primary jet as a result of the shear between them. It orients the jet slightly downwards, restraining the jet from spreading towards the upper kiln wall, and preventing possible thermal fatigue there. The ERZ entrains the passive scalar upwards, promoting the spreading of scalar in the spanwise direction. Moreover, the ERZ grows when Re increases. In a reactive case, the ERZ can improve the mixing between the reactants, assists in the flame anchoring, and hence further benefit the flame stability. Since a long jet stably located in the center of the kiln is expected in this type of kiln for iron ore pellets sintering, the existence of the ERZ is therefore significantly advantageous.

The $k-\varepsilon$ model can be improved for better accuracy but no matter how accurate it can predict the mean properties of the jet development, two equation models are still not capable of capturing the underlying flow structures such as vortex shedding and its effect on small-scale mixing. For better validation and understanding of the flow field in this virtual kiln model, more measurements and advanced turbulence models or methods will be considered in future work.

\section{Data Availability}

The data supporting the conclusions in this work are included in this manuscript. And the other datasets generated and/or analysed during the current study are available from the corresponding author on reasonable request.

\section{Conflicts of Interest}

The authors declare that they have no conflicts of interest.

\section{Acknowledgments}

The authors would like to acknowledge ASME 2016 International Mechanical Engineering Congress and Exposition (IMECE 2016) for presenting our prestudy to this work regarding the comparison of different turbulence models. Based on the conclusion from the prestudy, the work described in this manuscript can therefore be carried out. The authors would also like to acknowledge the meaningful discussions with LKAB, who also partly financed the work via the Faste Laboratory. This work was partly carried out within the framework of the Faste Laboratory, a VINNOVA Excellence Center for Functional Product Innovation and partly carried out within the VINNOVA STRIM-project "New digital 3D model of the Grate-Kiln pelletizing process for reduced energy usage and emissions" (2017-02170).

\section{References}

[1] S. Larsson, The Fluid Dynamics of the Cold Flow in a Rotary Kiln, Luleå University of Technology, Luleå, Sweden, 2014.

[2] I. A. S. Larsson, E. M. Lindmark, T. S. Lundström, and G. J. Nathan, "Secondary flow in semi-circular ducts," Journal of Fluids Engineering, vol. 133, no. 10, p. 101206, 2011.

[3] I. Larsson, E. M. Lindmark, T. S. Lundström, D. Marjavaara, and S. Töyrä, "Visualization of merging flow by usage of PIV and CFD with application to grate-kiln induration machines," Journal of Applied Fluid Mechanics (JAFM), vol. 5, no. 4, pp. 81-89, 2012.

[4] I. A. S. Larsson, B. R. Granström, T. S. Lundström, and B. D. Marjavaara, "PIV analysis of merging flow in a simplified model of a rotary kiln," Experiments in Fluids, vol. 53, no. 2, pp. 545-560, 2012.

[5] I. A. S. Larsson, T. S. Lundström, and B. D. Marjavaara, "Calculation of kiln aerodynamics with two RANS turbulence models and by DDES," Flow, Turbulence and Combustion, vol. 94, no. 4, pp. 859-878, 2015.

[6] I. A. Sofia Larsson, T. Staffan Lundström, and B. Daniel Marjavaara, "The flow field in a virtual model of a rotary kiln as a function of inlet geometry and momentum flux ratio," Journal of Fluids Engineering, vol. 137, no. 10, p. 101102, 2015.

[7] I. A. S. Larsson, B. D. Marjavaara, and T. S. Lundström, "Simulation of the flow field in an iron ore pelletizing kiln," 
Minerals and Metallurgical Processing, vol. 33, no. 3, pp. 144-148, 2016.

[8] I. A. S. Larsson, S. P. A. Johansson, T. S. Lundström, and B. D. Marjavaara, "PIV/PLIF experiments of jet mixing in a model of a rotary kiln," Experiments in Fluids, vol. 56, no. 5, 2015.

[9] Z. Teng, S. P. A. Johansson, I. A. S. Larsson, T. S. Lundström, and B. D. Marjavaara, "CFD simulation of jet mixing with asymmetric Co-flows in a down-scaled rotary kiln model," in Proceedings of the ASME 2016 International Mechanical Engineering Congress and Exposition, ASME, Phoenix, AZ, USA, November 2016.

[10] R. Granström, S. Lundström, D. Marjavaara, and S. Töyrä, "CFD modelling of the flow through a grate-kiln," in Proceedings from Seventh International Conference on Computational Fluid Dynamics in Minerals and Process Industries, Melbourne, VIC, Australia, December 2009.

[11] E. F. Winter, "1 - flow visualization techniques A2 - ducarme," in Progress in Combustion Science and Technology, M. Gerstein and A. H. Lefebvre, Eds., pp. 1-36, Pergamon Press, Oxford, UK, 1960.

[12] M. Glass and R. W. Bilger, "The turbulent jet diffusion flame in a Co-flowing stream-some velocity measurements," Combustion Science and Technology, vol. 18, no. 5-6, pp. 165-177, 1978.

[13] D. C. Guío-Pérez, G. Tondl, W. Höltl, T. Pröll, and H. Hofbauer, "Cold flow model study of an oxyfuel combustion pilot plant," Chemical Engineering and Technology, vol. 34, no. 12, pp. 2091-2098, 2011.

[14] S. Corrsin, Investigation of Flow in an Axially Symmetrical Heated Jet of Air, National Advisory Committee for Aeronautics, Washington, DC, USA, 1943.

[15] H. W. Liepmann and J. Laufer, Investigations of Free Turbulent Mixing, NACA-TN-1257, National Advisory Committee for Aeronautics, Washington, DC, USA, 1947.

[16] G. N. Faris, Some Entrainment Properties of a Turbulent AxiSymmetric Jet, Aerophysics Department, Mississippi State University, Starkville, MS, USA, 1963.

[17] I. Wygnanski and H. Fiedler, Some Measurements in the Self Preserving Jet, Cambridge University Press, Cambridge, UK, 1968.

[18] H. J. Hussein, S. P. Capp, and W. K. George, "Velocity measurements in a high-Reynolds-number, momentumconserving, axisymmetric, turbulent jet," Journal of Fluid Mechanics, vol. 258, no. 1, pp. 31-75, 1994.

[19] R. A. Antonia and R. W. Bilger, "An experimental investigation of an axisymmetric jet in a Co-flowing air stream," Journal of Fluid Mechanics, vol. 61, no. 4, pp. 805-822, 1973.

[20] F. H. Champagne and I. J. Wygnanski, "An experimental investigation of coaxial turbulent jets," International Journal of Heat and Mass Transfer, vol. 14, no. 9, pp. 1445-1464, 1971.

[21] W. K. George, "The self-preservation of turbulent flows and its relation to initial conditions and coherent structures," in Advances in Turbulence, W. K. George and R. Arndt, Eds., pp. 39-73, Springer, Berlin, Germany, 1989.

[22] B. J. Boersma, G. Brethouwer, and F. T. M. Nieuwstadt, "A numerical investigation on the effect of the inflow conditions on the self-similar region of a round jet," Physics of Fluids, vol. 10, no. 4, pp. 899-909, 1998.

[23] J. Mi, D. S. Nobes, and G. J. Nathan, "Influence of jet exit conditions on the passive scalar field of an axisymmetric free jet," Journal of Fluid Mechanics, vol. 432, pp. 91-125, 2001.

[24] V. Todde, P. G. Spazzini, and M. Sandberg, "Experimental analysis of low-Reynolds number free jets," Experiments in Fluids, vol. 47, no. 2, pp. 279-294, 2009.
[25] P. Burattini, R. A. Antonia, and L. Danaila, "Similarity in the far field of a turbulent round jet," Physics of Fluids, vol. 17, no. 2, p. 025101, 2005.

[26] T. H. Weisgraber and D. Liepmann, "Turbulent structure during transition to self-similarity in a round jet," Experiments in Fluids, vol. 24, no. 3, pp. 210-224, 1998.

[27] D. Mistry and J. Dawson, "Experimental investigation of multi-scale entrainment processes of a turbulent jet," in Proceedings of 17th International Symposium on Application of Laser Techniques to Fluid Mechanics, Munich, Germany, 2014.

[28] A. M. Falcone and J. C. Cataldo, "Entrainment velocity in an axisymmetric turbulent jet," Journal of Fluids Engineering, vol. 125 , no. 4, pp. 620-627, 2003.

[29] G. A. Zarruk and E. A. Cowen, "Simultaneous velocity and passive scalar concentration measurements in low Reynolds number neutrally buoyant turbulent round jets," Experiments in Fluids, vol. 44, no. 6, pp. 865-872, 2008.

[30] A. J. Yule and M. Damou, "Investigations of ducted jets," Experimental Thermal and Fluid Science, vol. 4, no. 4, pp. 469-490, 1991.

[31] E. Smith, G. Nathan, and B. Dally, Range of Validity of a Modified K-Epsilon Model of the Non-Reacting Flow from a Precessing Jet Nozzle, CSIRO, Canberra, NSW, Australia, 2003.

[32] X. Grandchamp, Y. Fujiso, B. Wu, and A. V. Hirtum, "Steady laminar axisymmetrical nozzle flow at moderate Reynolds numbers: modeling and experiment," Journal of Fluids Engineering, vol. 134, no. 1, p. 011203, 2012.

[33] Y. Tominaga and T. Stathopoulos, "Turbulent Schmidt numbers for CFD analysis with various types of flowfield," Atmospheric Environment, vol. 41, no. 37, pp. 8091-8099, 2007.

[34] J. M. Mejía, A. Sadiki, A. Molina, F. Chejne, and P. Pantangi, "Large eddy simulation of the mixing of a passive scalar in a high-Schmidt turbulent jet," Journal of Fluids Engineering, vol. 137, no. 3, p. 031301, 2015.

[35] B. E. Launder and B. I. Sharma, "Application of the energydissipation model of turbulence to the calculation of flow near a spinning disc," International Communications in Heat and Mass Transfer, vol. 1, no. 2, pp. 131-137, 1974.

[36] I. Celik, "Procedure for estimation and reporting of uncertainty due to discretization in CFD applications," Journal of Fluids Engineering, vol. 130, no. 7, p. 078001, 2008.

[37] J. F. Driscoll, R. H. Chen, and V. Tangirala, "The role of recirculation in improving internal mixing and stability of flames," AIAA Journal, vol. 87, pp. 306-325, 1987.

[38] K. P. Vanoverberghe, E. V. Van Den Bulck, and M. J. Tummers, "Confined annular swirling jet combustion," Combustion Science and Technology, vol. 175, no. 3, pp. 545-578, 2003.

[39] M. Rabacal, B. M. Franchetti, F. C. Marincola et al., "Large Eddy Simulation of coal combustion in a large-scale laboratory furnace," in Proceedings of the Combustion Institute, vol. 35, no. 3, pp. 3609-3617, 2015.

[40] E. J. Smith, J. Mi, G. J. Nathan, and B. B. Dally, "Preliminary examination of a "round jet initial condition anomaly" for the $k$ - $\varepsilon$ turbulence model," in Proceedings of 15th Australasian Fluid Mechanics Conference, Sydney, NSW, Australia, December 2004.

[41] S. B. Pope, "An explanation of the turbulent round-jet/planejet anomaly," AIAA Journal, vol. 16, no. 3, pp. 279-281, 1978.

[42] Z. Teng, "CFD Simulation of jet in asymmetric Co-flows in a down-scaled rotary kiln model," Licentiate thesis, Luleå University of Technology, Luleå, Sweden, 2018. 


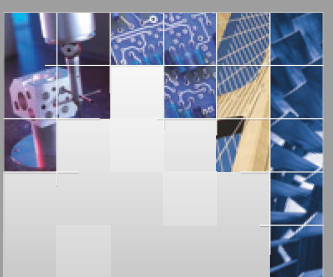

\section{Enfincering}
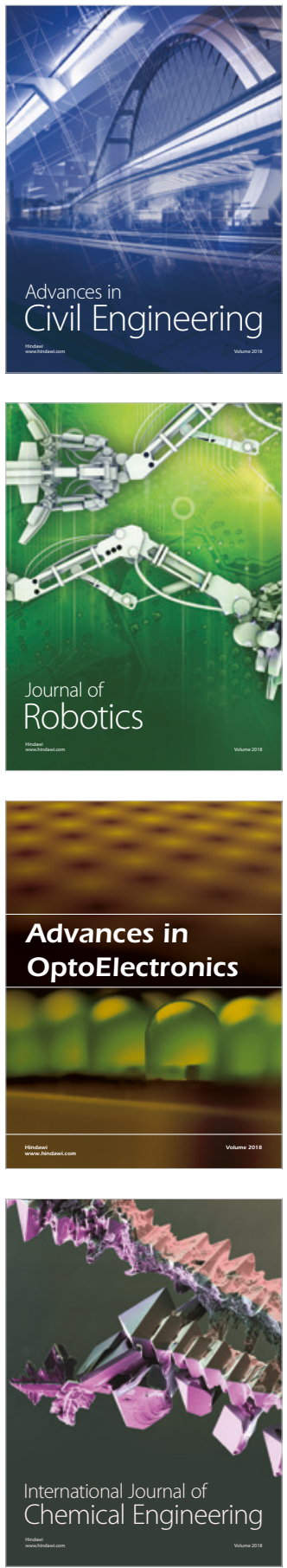

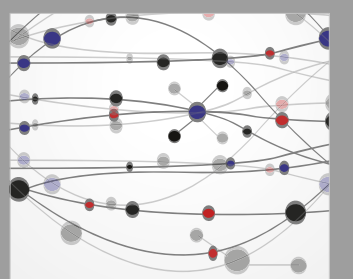

\section{Rotating \\ Machinery}

The Scientific World Journal

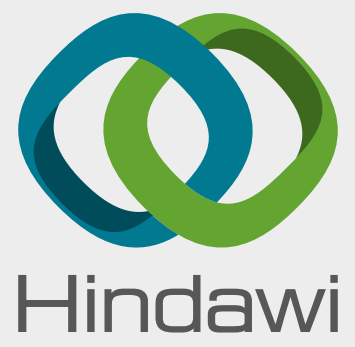

Submit your manuscripts at

www.hindawi.com
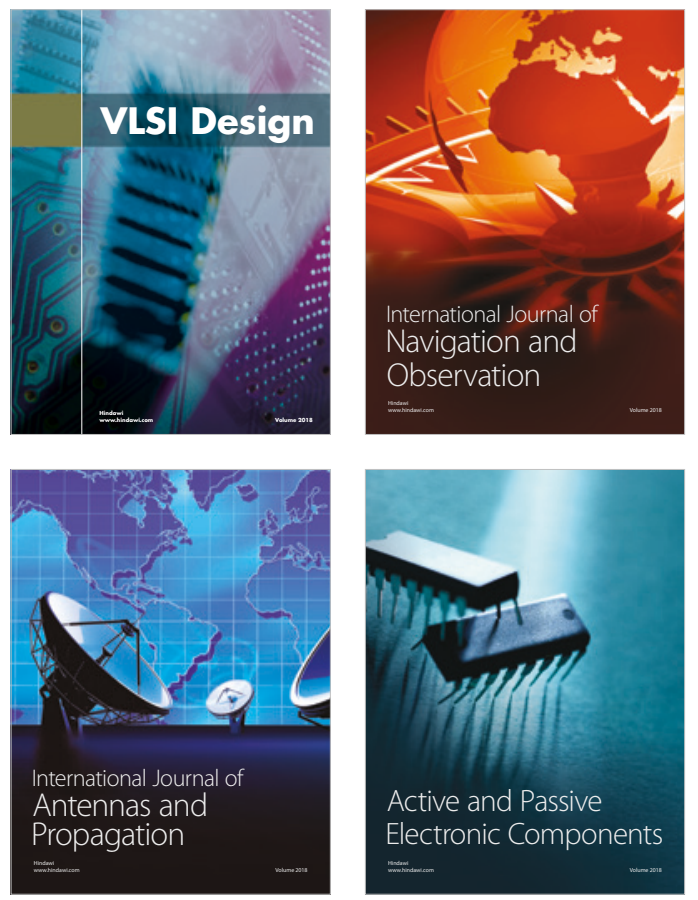
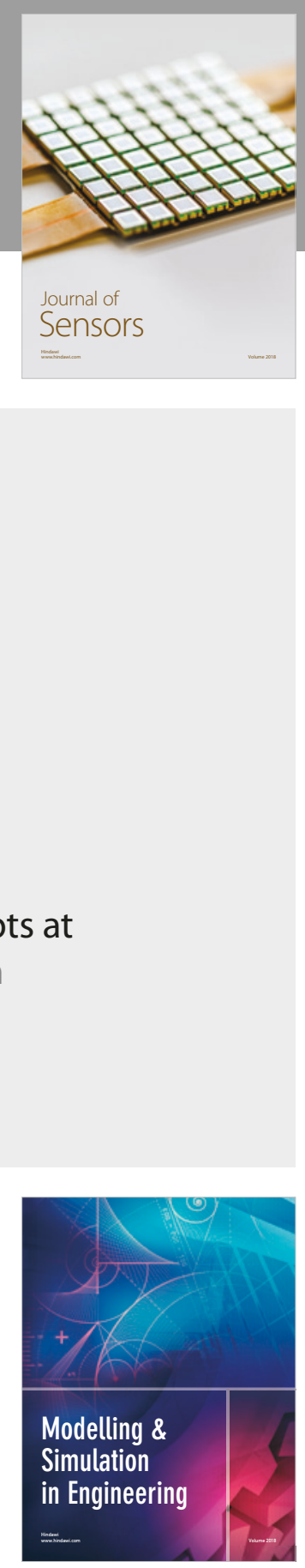

\section{Advances \\ Multimedia}
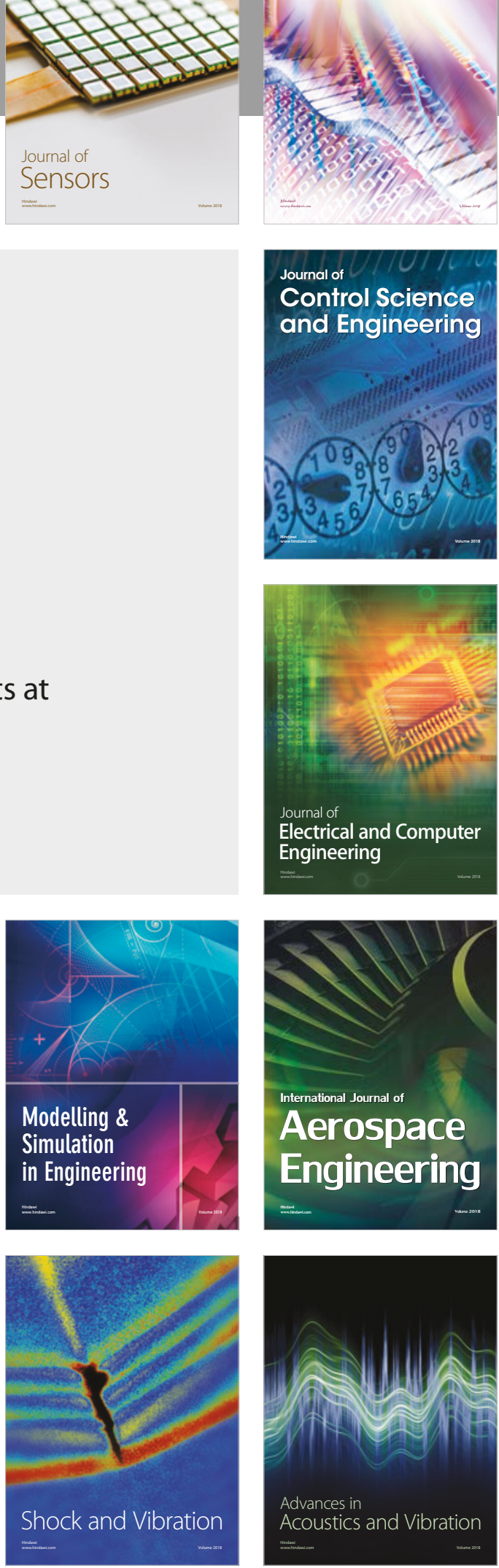